Urologe $2021 \cdot 60: 351-360$

https://doi.org/10.1007/s00120-021-01444-5

Angenommen: 4. Januar 2021

Online publiziert: 22. Januar 2021

๑ Der/die Autor(en) 2021

\section{()}

Check for
updates

Martin Baunacke ${ }^{1}$ Christer Groeben ${ }^{1}$ Angelika Borkowetz Annemarie Uhlig $^{2}$ • Marianne Leitsmann ${ }^{2} \cdot$ Björn Volkmer ${ }^{3}$. Christian Thomas ${ }^{1} \cdot$ Johannes Huber ${ }^{1}$

' Klinik und Poliklinik für Urologie, Universitätsklinikum Carl Gustav Carus an der Technischen Universität Dresden, Dresden, Deutschland

${ }^{2}$ Klinik für Urologie, Universitätsmedizin Göttingen, Georg-August-Universität, Göttingen, Deutschland

${ }^{3}$ Klinik für Urologie, Klinikum Kassel, Kassel, Deutschland

\title{
Versorgungswirklichkeit der urologischen Endoprothetik in Deutschland von 2006 bis 2016
}

besondere bei Frauen erfolgt die Anlage um den Blasenhals. Die Sphinkterprothese stellt den Goldstandard bei der Therapie der männlichen Belastungsinkontinenz nach radikaler Prostatektomie (RP) dar und erreicht hier hohe Erfolgsraten zwischen 59-90\% [1-3]. Nachteile der Sphinktersysteme sind die nicht zu vernachlässigenden Komplikations- und Revisionsraten sowie die begrenzte Haltbarkeit [4]. Die Revisionsrate sinkt mit zunehmender operativer Erfahrung [5].

Penisprothesen sind Schwellkörperimplantate und finden ihren Einsatz nach zahlreichen Therapiemöglichkeiten der erektilen Dysfunktion wie oraler und urethraler Pharmakotherapie, Schwellkörperautoinjektion oder Vakuumpenispumpen. Ein weiterer relevanter Anwendungsbereich ist der Einsatz in der operativen Geschlechtsangleichung. In kleinerer Zahl werden Penisprothesen auch im Rahmen von Penisrekonstruktionen nach Amputationen bei Unfällen oder Peniskarzinomen implantiert [6]. Bei Penisprothesen wird zwischen semirigiden und hydraulischen Prothesen unterschieden. Trotz der Radikalität des Verfahrens ist die Penisprothese der Goldstandard nach Versagen konservativer Therapien. In diesem Patientenkollektiv mit hohem Leidensdruck erreicht die Penisprothese deutlich höheren Zufriedenheitsraten als die Pharmakotherapie [1]. Auch die Penisprothesenimplantation weißt eine relevante Komplikationsrate auf [7], wel- che durch operative Erfahrung gesenkt werden kann [8].

Trotz guter Erfolgsraten von Sphinkter- und Penisprothesen konnten wir aktuell Anhaltspunkte für ein relevantes Versorgungsdefizit in der Behandlung von Patienten mit Inkontinenz bzw. erektiler Dysfunktion nach RP in Deutschland finden [9]. Daher ist es Ziel dieser Studie, die Versorgungswirklichkeit der Sphinkter- und Penisprothesenimplantation in Deutschland zu analysieren.

\section{Material und Methoden}

Wir führten eine Analyse der Diagnosis Related Groups(DRG)-Abrechnungsdaten in Deutschland im Zeitraum von 2006 bis 2016 über das Statistische Bundesamt (Destatis) durch. Hierbei erfolgte für Sphinkterprothesen die Abfrage der jährlichen Patientenzahl für folgende Operationen- und Prozedurenschlüssel(OPS)Codes: 5-597-00 (Implantation bulbär, 1 Cuff), 5-597-01 (Implantation bulbär, 2 Cuffs) und 5-597-02 (Implantation am Blasenhals). Für den OPS-Code 5-597-0 (Implantation) erfolgte zusätzlich die Abfrage der Zahl der abrechnenden Kliniken und des Patientenalters. Für Penisprothesen erfolgte die Analyse folgender OPS-Codes: 5-649-5 (Implantation einer Penisprothese), 5-649-50 (Implantation semirigide Prothese) und 5-64951 (Implantation hydraulische Prothese). Für die OPS 5-649-5 wurden zusätzlich die Zahl der abrechnenden Kliniken, das Patientenalter und die Verknüpfung mit
Qualität und Ökonomie" der DGU e.V. und der Arbeitsgruppe "Versorgungsforschung \& Soziale Medien" der GeSRU Academics. 


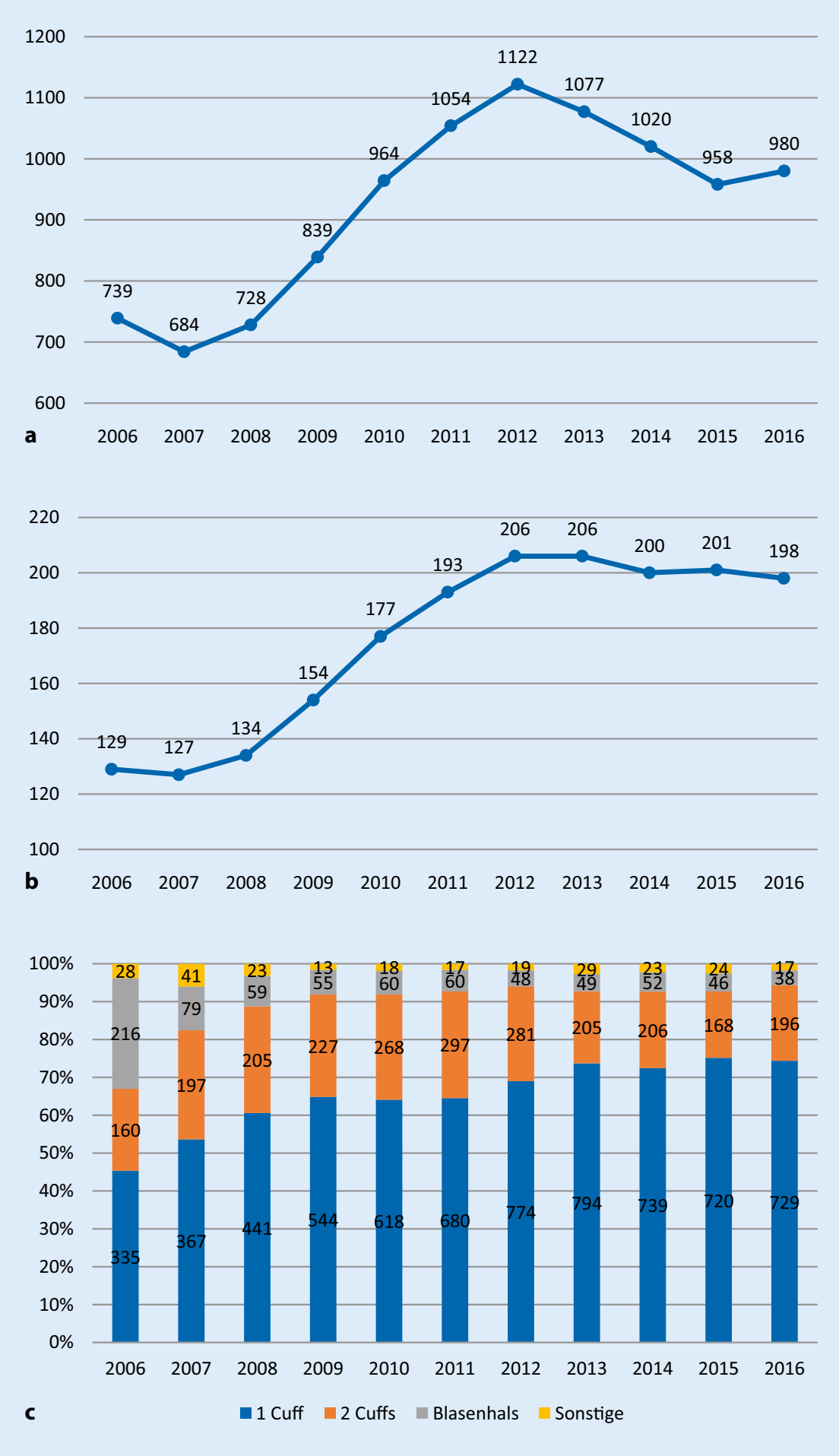

Abb. $1 \Delta$ a Zahl der implantierten Sphinkterprothesen. b Zahl der urologischen Kliniken, die Sphinkterprothesen implantierten. c Zahl der implantierten Sphinkterprothesentypen

der ICD(Internationale statistische Klassifikation der Krankheiten) F64 (Störung der Geschlechtsidentität) ermittelt. Zur weiteren Einordnung der Fallzahlen erfolgte die Abfrage der OPS 5-604* (radikale Prostatovesikulektomie) [10]. der Software QB-Monitor 2016 (Lutum+Tappert DV-Beratung GmbH, Bonn). Aus Datenschutzgründen wird in den QB-Daten zu ICD- und OPSKennziffern mit einer Anzahl von 1, 2 oder 3 die tatsächliche Zahl nicht ausgewiesen. Im QB-Monitor wird in diesen Fällen die Zahl 1 angegeben. Die ermittelten Kliniken wurden bezüglich der Bettenzahl des Krankenhauses, der Bettenzahl der urologischen Klinik, der Stadtgröße und der RP-Fallzahl klassifiziert. Außerdem ergänzten wir, ob es sich um eine Universitätsklinik und ein zertifiziertes Prostatakarzinomzentrum handelte. Bei der Sphinkterprothesenimplantation erfolgte folgende Einteilung: Kliniken mit geringer Fallzahl (1-9 Sphinkterprothesen) und hoher Fallzahl ( $\geq 10$ Sphinkterprothesen). Bei der Penisprothesenimplantation erfolgte folgende Einteilung: Kliniken mit geringer Fallzahl (1-6 Penisprothesen) und hoher Fallzahl ( $\geq 7$ Penisprothesen). Die 10 Top-Kliniken bei Sphinkterprothesen implantierten $\geq 20$ Sphinkterprothesen 2016. Die 7 Top-Kliniken bei Penisprothesen implantierten $\geq 20$ Penisprothesen 2016. Die Kartendarstellungen erfolgten mit der Software easymap $\odot$ office (Lutum + Tappert DV-Beratung $\mathrm{GmbH}$, Bonn).

$\mathrm{Da}$ alle verwendeten Daten anonymisiert sind, war kein Ethikvotum erforderlich. Die Datenanalyse erfolgte mit dem $\chi^{2}$-Test, Mann-Whitney-U-Test und linearer Regressionsanalyse (Signifikanzniveau $p=0,05$ ). Die statistische Auswertung erfolgte mit „IBM SPSS Statistics $27^{\text {“ }}$ (Armonk, NY, USA).

\section{Ergebnisse}

\section{Sphinkterprothetik}

Von 2006 bis 2012 zeigte sich ein kontinuierlicher Anstieg der implantierenden Kliniken $(+15 / \mathrm{Jahr}, p<0,001)$ und der Gesamtfallzahl $(+76 / \mathrm{Jahr}, p<0,001)$. Ab 2012 nahm die Zahl der Implantationen von 1122 auf 980 Implantationen ab $(-40 / J a h r, p=0,02)$ und die Anzahl der Kliniken sank von 206 auf 198 $(-2 / \mathrm{Jahr}, p=0,03)$. Der Anteil an Frauen bei Sphinkterimplantationen blieb zwischen 2006 bis 2016 mit 2-5\% kon- 
Urologe 2021·60:351-360 https://doi.org/10.1007/s00120-021-01444-5

(c) Der/die Autor(en) 2021

\section{Baunacke · C. Groeben · A. Borkowetz · A. Uhlig · M. Leitsmann · B. Volkmer · C. Thomas · J. Huber}

\section{Versorgungswirklichkeit der urologischen Endoprothetik in Deutschland von 2006 bis 2016}

\section{Zusammenfassung}

Hintergrund. Die Behandlung von Harninkontinenz und erektiler Dysfunktion verbessert die Lebensqualität vieler Patienten. Insbesondere die Endoprothetik mit Sphinkterund Penisprothesen erzielt hierbei sehr gute Ergebnisse, wenn konservative Therapieoptionen ausgeschöpft sind. Ziel dieser Studie ist eine Darstellung der Entwicklung und aktuellen Versorgungslage der Sphinkter- und Penisprothesenimplantation in Deutschland. Material und Methoden. Wir führten eine Analyse der Diagnosis-Related-GroupsAbrechnungsdaten in Deutschland im Zeitraum von 2006 bis 2016 durch. Die Versorgungslage im Jahr 2016 beschrieben wir auf Basis der Qualitätsberichtsdaten der deutschen Krankenhäuser.

Ergebnisse. Von 2006 bis 2012 stieg die Zahl der implantierten Sphinkterprothesen in Deutschland von 739 auf $1112(p<0,001)$ und die Zahl der implantierenden Kliniken von 129 auf $206(p<0,001)$. Von 2012 bis 2016 fielen die Fallzahlen auf 980 und die Zahl der Kliniken auf 198. Im Jahr 2016 implantierten 168 (88\%) urologische Kliniken 1-9 Sphinkterprothesen und 23 (12\%) Kliniken $\geq 10$ Sphinkterprothesen. Die 10 TopKliniken ( $\geq 20$ Sphinkter) implantierten $34 \%$ (283/839) aller Sphinkter. Von 2006 bis 2013 stieg die Zahl der implantierten Penisprothesen kontinuierlich von 263 auf $503(p<0,001)$ sowie die Zahl der implantierenden Kliniken von 71 auf 107 $(p<0,001)$. Von 2013 bis 2016 stagnierte die Fallzahl $(p=0,9)$ und die Zahl der implantierenden Kliniken $(p=0,5)$. Der Anteil implantierter Penisprothesen im Rahmen von Geschlechtsumwandlungen stieg von $17 \%$ im Jahr 2006 auf $25 \%$ im Jahr $2016(p=0,03)$. Im Jahr 2016 implantierten 83 (85\%) urologische
Kliniken 1-6 Penisprothesen und 14 (15\%) Kliniken $\geq 7$ Prothesen. Die 7 Top-Kliniken ( $\geq 20$ Prothesen/Jahr) implantierten 232/448 (52\%) der Prothesen.

Diskussion. Der Versorgungsstand der urologischen Endoprothetik in Deutschland zeigt eine deutliche Zentrenbildung, aber auch eine große Zahl von Kliniken mit geringer Fallzahl. Seit 2012/2013 zeigt sich eine Stagnation der Fallzahlen von Penisund Sphinkterprothesenimplantationen, die in Zusammenschau mit den Prostatektomiefallzahlen eine Unterversorgung vermuten lässt.

\section{Schlüsselwörter}

Sphinkterprothese - Penisprothese . Inkontinenz - Erektile Dysfunktion . Versorgung

\section{Health care reality of urological endoprosthetics in Germany from 2006 to 2016}

\section{Abstract}

Background. Treating urinary incontinence and erectile dysfunction improves quality of life for many patients. In particular, sphincter and penile prostheses achieve very good results when conservative therapy options are exhausted. The aim of this study is to present the development and current state of sphincter and penile prosthesis implantation in Germany.

Materials and methods. We carried out an analysis of the Diagnosis Related Groups billing data in Germany from 2006-2016. We described the state of care in 2016 based on the German hospitals' quality reports.

Results. Between 2006 and 2012 implantations of sphincter prostheses in Germany increased from 739 to $1112(p<0.001)$, the amount of implanting hospitals also increased from 129 to $206(p<0.001)$. From 2012-2016, the number of cases decreased to 980 and the number of hospitals to $198 . \ln 2016,168$ (88\%) urological hospitals implanted 1-9 sphincter prostheses and 23 (12\%) hospitals implanted $\geq 10$ sphincter prostheses. The top 10 hospitals ( $\geq 20$ sphincters) implanted $34 \%$ (283/839) of all sphincters. Between 2006 and 2013 the number of implanted penile prostheses continuously increased from 263 to $503(p<0.001)$, the number of implanting hospitals from 71 to $107(p<0.001)$. From 2013-2016, the number of cases $(p=0.9)$ and the number of implanting hospitals $(p=0.5)$ stagnated. The proportion of penile prostheses implanted as part of gender reassignment surgery increased from $17 \%$ in 2006 to $25 \%$ in $2016(p=0.03)$. In 2016, $83(85 \%)$ urological hospitals implanted 1-6 penile prostheses and $14(15 \%)$ hospitals implanted $\geq 7$ prostheses. The 7 top hospitals ( $\geq 20$ prostheses/year) implanted 232/448 (52\%) of the prostheses.

Conclusions. The current state of urological endoprosthetics in Germany shows a small number of high-volume centers, but also a large number of hospitals with a small number of cases. Since 2012/2013, there has been a stagnation in the number of cases of penile and sphincter prosthesis implantations. In view of the number of radical prostatectomy cases, this development suggests an undersupply.

\section{Keywords}

Sphincter prosthesis - Penile prosthesis . Incontinence - Erectile dysfunction · Health care research stant. Der Anteil von Sphinktern mit einem Cuff nahm von 2006 bis 2016 von $45 \%(335 / 739)$ auf $74 \%(729 / 980)$ zu $(p<0,001)$, während der Anteil der Doppel-Cuff-Prothesen leicht sank - von $22 \%(160 / 739) 2006$ bzw. $29 \%(197 / 684)$ 2007 auf $20 \%(196 / 980) 2016(p=0,02)$. Auch der Anteil von Sphinktern mit Blasenhalsmanschette sank deutlich von
$29 \%(216 / 739)$ auf $4 \%(38 / 980 ; p=0,02$; - Abb. 1). Der Anteil männlicher Patienten bei der Implantation von Sphinktern mit Blasenhalsmanschette nahm ebenfalls signifikant ab von $82 \%(177 / 216)$ auf $45 \%(17 / 38 ; p<0,001)$, während der absolute Anteil an Frauen mit jeweils 21-39 Frauen pro Jahr konstant blieb. Es zeigte sich eine signifikante Zunah- me im Durchschnittsalter der Patienten im Zeitraum von 2006 bis 2016 von 67,9 Jahren auf 70,7 Jahre $(p<0,001)$.

In Deutschland wurden 2016839 Sphinkterprothesen in 191 urologischen Kliniken implantiert (- Tab. 1 und - Abb. 2). 13 Sphinkterprothesen wurden jeweils durch zwei chirurgische und zwei gynäkologische Kliniken implan- 


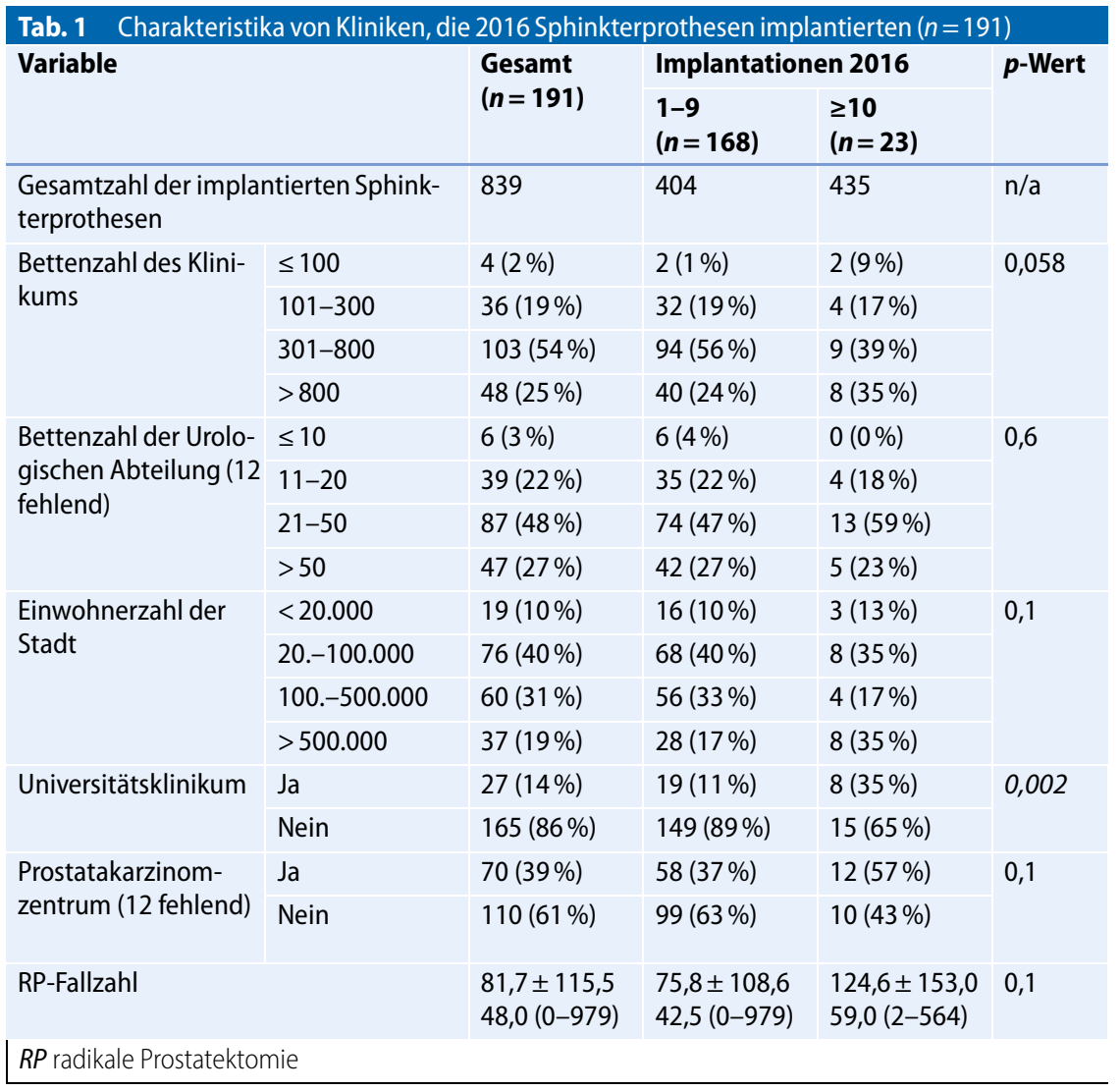

\begin{tabular}{|c|c|c|c|c|c|}
\hline \multirow{2}{*}{\multicolumn{2}{|c|}{ Variable }} & \multirow{2}{*}{$\begin{array}{l}\text { Gesamt } \\
(n=97)\end{array}$} & \multicolumn{2}{|c|}{ Implantationen 2016} & \multirow[t]{2}{*}{$p$-Wert } \\
\hline & & & $\begin{array}{l}1-6 \\
(n=83)\end{array}$ & $\begin{array}{l}>6 \\
(n=14)\end{array}$ & \\
\hline \multicolumn{2}{|c|}{$\begin{array}{l}\text { Zahl der implantierten Penisprothe- } \\
\text { sen }\end{array}$} & 448 & 144 & 304 & $n / a$ \\
\hline \multirow{4}{*}{$\begin{array}{l}\text { Bettenzahl des Kli- } \\
\text { nikum ( } 3 \text { fehlend) }\end{array}$} & $\leq 100$ & $2(2 \%)$ & $1(1 \%)$ & $1(8 \%)$ & \multirow[t]{4}{*}{0,09} \\
\hline & $101-300$ & $14(15 \%)$ & $13(16 \%)$ & $1(8 \%)$ & \\
\hline & $301-800$ & $45(48 \%)$ & $42(51 \%)$ & $3(25 \%)$ & \\
\hline & $>800$ & $33(35 \%)$ & $26(32 \%)$ & $7(59 \%)$ & \\
\hline \multirow{4}{*}{$\begin{array}{l}\text { Bettenzahl der } \\
\text { Urologischen } \mathrm{Ab} \text { - } \\
\text { teilung ( } 5 \text { fehlend) }\end{array}$} & $\leq 10$ & $3(3 \%)$ & $3(4 \%)$ & $0(0 \%)$ & \multirow[t]{4}{*}{0,5} \\
\hline & $11-20$ & $16(18 \%)$ & $15(19 \%)$ & $1(8 \%)$ & \\
\hline & $21-50$ & $49(53 \%)$ & $43(53 \%)$ & $6(50 \%)$ & \\
\hline & $>50$ & $24(26 \%)$ & $19(24 \%)$ & $5(42 \%)$ & \\
\hline \multirow{4}{*}{$\begin{array}{l}\text { Einwohnerzahl der } \\
\text { Stadt }\end{array}$} & $<20.000$ & $10(10 \%)$ & $10(12 \%)$ & $0(0 \%)$ & \multirow[t]{4}{*}{0,001} \\
\hline & 20.-100.000 & $27(28 \%)$ & $25(30 \%)$ & $2(14 \%)$ & \\
\hline & 100.-500.000 & $38(39 \%)$ & $35(42 \%)$ & $3(22 \%)$ & \\
\hline & $>500.000$ & $22(23 \%)$ & $13(16 \%)$ & $9(64 \%)$ & \\
\hline \multirow{2}{*}{$\begin{array}{l}\text { Universitäts- } \\
\text { klinikum }\end{array}$} & Ja & $23(24 \%)$ & $16(19 \%)$ & $7(50 \%)$ & \multirow[t]{2}{*}{0,01} \\
\hline & Nein & $74(76 \%)$ & $67(81 \%)$ & $7(50 \%)$ & \\
\hline \multirow{2}{*}{$\begin{array}{l}\text { Prostatakarzinom- } \\
\text { zentrum ( } 5 \text { feh- } \\
\text { lend) }\end{array}$} & Ja & $32(35 \%)$ & $28(35 \%)$ & $4(33 \%)$ & \multirow[t]{2}{*}{0,9} \\
\hline & Nein & $60(65 \%)$ & $52(65 \%)$ & $8(67 \%)$ & \\
\hline \multicolumn{2}{|l|}{ RP-Fallzahl } & $\begin{array}{l}110,6 \pm 151,4 \\
55,5(0-979)\end{array}$ & $\begin{array}{l}105,0 \pm 148,7 \\
55,5(0-979)\end{array}$ & $\begin{array}{l}143,3 \pm 168,2 \\
74,5(2-486)\end{array}$ & 0,7 \\
\hline \multicolumn{2}{|c|}{$\begin{array}{l}\text { Fallzahl mit Störung der Ge- } \\
\text { schlechtsidentität (ICD F64) }\end{array}$} & $\begin{array}{l}7,6 \pm 44,3 \\
0(0-415)\end{array}$ & $\begin{array}{l}1,7 \pm 12,6 \\
0(0-113)\end{array}$ & $\begin{array}{l}42,2 \pm 109,5 \\
0,5(0-415)\end{array}$ & $<0,001$ \\
\hline \multicolumn{5}{|c|}{$R P$ radikale Prostatektomie } & \\
\hline
\end{tabular}

tiert. 168 (88\%) der urologischen Kliniken implantierten 1-9 Sphinkterprothesen, 23 (12\%) Kliniken implantierten $\geq 10$ Sphinkterprothesen. Kliniken mit geringer Fallzahl (1-9) implantierten $48 \%$ (404/839) der Sphinkter. Kliniken mit hoher Fallzahl $(\geq 10)$ implantierten $52 \%$ (435/839) der Sphinkter. Die 10 TopKliniken ( $\geq 20$ Sphinkter) implantierten $34 \%(283 / 839)$ aller Sphinkter. Kliniken mit einer Fallzahl $\geq 10$ Sphinkterprothesen/Jahr sind häufiger Universitätskliniken ( $35 \%$ vs. $11 \%, p=0,002)$. Es gibt keinen signifikanten Zusammenhang zur Größe der urologischen Klinik $(p=0,6)$, der Stadtgröße $(p=0,1)$ und der jährlichen RP-Fallzahl ( $p=0,1$; • Tab. 1).

\section{Penisprothetik}

Von 2006 bis 2013 zeigt sich ein kontinuierlicher Anstieg der implantierten Penisprothesen von 263 auf $503(+33 /$ Jahr, $p<0,001)$, sowie ein Anstieg der implantierenden Kliniken von 71 auf 107 (+5/Jahr, $p<0,001)$. Ab 2013 bis 2016 stagnierten die Fallzahl $(p=0,9)$ und die Zahl der implantierenden Kliniken $(p=0,5)$. Der Anteil hydraulischer Prothesen nahm von 2006 bis 2016 von $85 \%(223 / 263)$ auf $96 \%(505 / 526) \mathrm{zu}$ $(p=0,02)$, während der Anteil der semirigiden Prothesen von $13 \%(35 / 263)$ auf $4 \%(21 / 526)$ sank $(p=0,002)$. Weiterhin nahm der Anteil implantierter Penisprothesen im Rahmen von Geschlechtsumwandlungen von $17 \%$ (45/263) im Jahr 2006 auf $25 \%(130 / 526)$ im Jahr $2016 \mathrm{zu}(p=0,03$; D Abb. 3). Es zeigte sich keine signifikante Entwicklung im Durchschnittsalter der Patienten von 2006 bis $2016(p=0,9)$.

2016 wurden 448 Penisprothesen in 97 urologischen Kliniken in Deutschland implantiert (•Tab. 2 und $\bullet$ Abb. 4). 45 Penisprothesen wurden durch 6 chirurgische Kliniken implantiert. 83 (86\%) der urologischen Kliniken implantierten 1-6 Penisprothesen, 7 (7\%) Kliniken implantierten 7-20 Prothesen, 6 (6\%) Kliniken 21-32 Prothesen und 1 (1\%) Klinik 71 Penisprothesen. Kliniken mit geringer Fallzahl $(\leq 6)$ implantierten 144/448 (32\%) Penisprothesen und Kliniken mit hoher Fallzahl $(>6)$ implantierten 304/448 (68\%) Penisprothesen. 


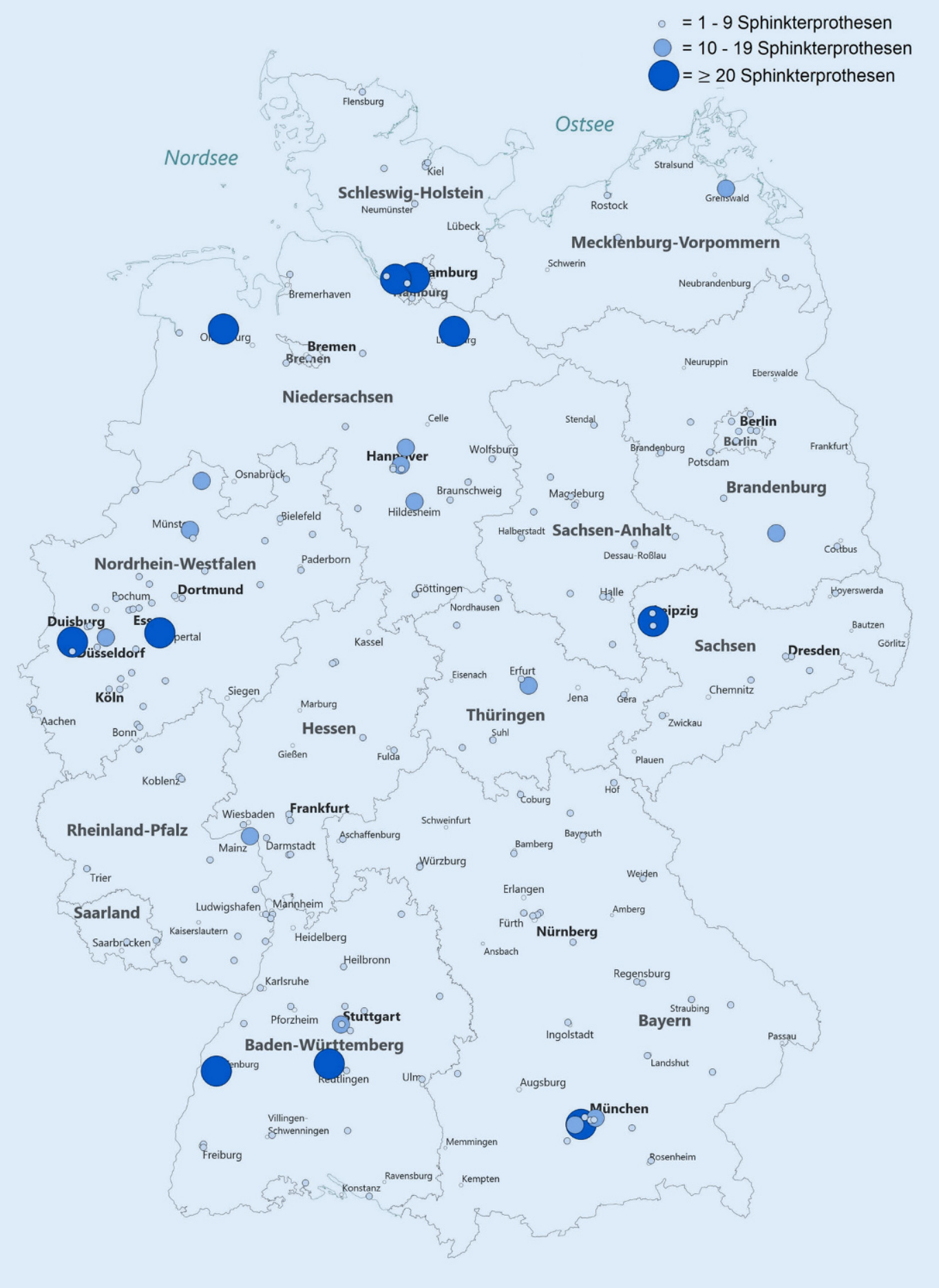

Abb. 2 < Urologische Kliniken, welche 2016 Implantationen von Sphinkterprothesen durchführten (Datenquelle: Qualitätsberichtsdaten 2016, g-ba; easymap@ Kartengrafik: Eigene Darstellung)

Die 7 Top-Kliniken ( $\geq 20$ Prothesen/ Jahr) implantierten 232/448 (52\%) der Prothesen. Kliniken mit einer Fallzahl $>6$ Penisprothesen/Jahr sind häufiger Universitätskliniken $(50 \%$ vs. $19 \%$, $p=0,01)$, liegen häufiger in Großstädten ( $64 \%$ vs. $16 \%, p=0,001$ ) und haben eine höhere Fallzahl an Patienten mit Störung der Geschlechtsidentität (42,2 $\pm 109,5$ vs.
$1,7 \pm 12,6$; $p<0,001)$. Es gibt keinen signifikanten Zusammenhang zur Größe der urologischen Klinik $(p=0,5)$ und jährlichen RP-Fallzahl ( $p=0,7$; •Tab. 2).

Zur besseren Einordnung eines möglichen Versorgungsdefizits zeigt • Abb. 5 die jährlichen Fallzahlen der RP in Deutschland von 2006 bis 2016.

\section{Diskussion}

Bei der Implantation von Sphinkterprothesen zeigte sich von 2006 bis 2012 ein kontinuierlicher Anstieg der Gesamtfallzahl $(+76 / \mathrm{Jahr}, p<0,001)$ und der implantierenden Kliniken $(+15 / \mathrm{Jahr}$, $p<0,001)$. Seit 2012 nahm die Fallzahl $(-40 / \mathrm{Jahr}, p=0,02)$ und die Klinikzahl 


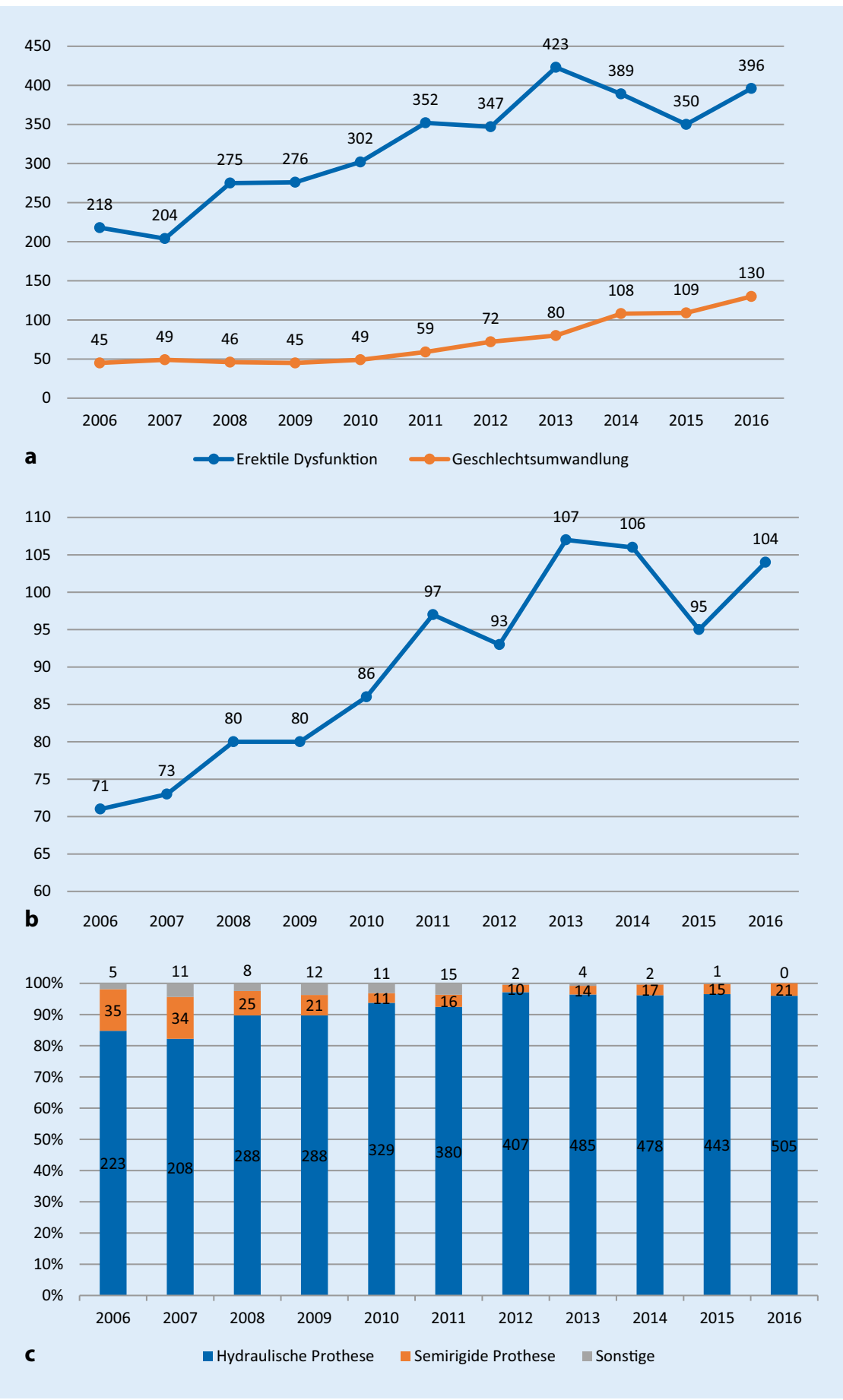

Abb. $3 \Delta$ a Zahl der implantierten Penisprothesen. b Zahl der urologischen Kliniken, die Penisprothesen implantierten. c Zahl der implantierten Penisprothesentypen

$(-2 / \mathrm{Jahr}, p=0,03)$ ab. Die 10 Top-Kliniken ( $\geq 20$ Sphinkter) implantierten ein Drittel aller 839 Sphinkter. Es zeigte sich ein deutlicher Abfall des Einsatzes von Sphinktern mit Blasenmanschetten von $29 \%$ auf $4 \%$ aufgrund des geringeren Einsatzes bei Männern bei gleichbleibenden Implantationszahlen bei Frauen.
Geschlechtsumwandlungen nahm kontinuierlich von 2006 bis $2016 \mathrm{zu}(p=0,03)$. Die 7 Top-Kliniken ( $\geq 20$ Prothesen/Jahr) implantierten die Hälfte aller 448 Prothesen. Es gab bei der Fallzahl der Sphinkterprothesen und der Penisprothesen keinen Zusammenhang mit der Fallzahl der radikalen Prostatektomien der jeweiligen Klinik ( $p=0,1$ bzw. $p=0,7)$.

Stressinkontinenz und erektile Dysfunktion nach RP stellen die häufigsten Indikationen für die Implantation von Sphinkter- und Penisprothese dar. Laut der großen deutschen Versorgungsforschungsstudie HAROW (Hormontherapie, Active Surveillance, Radiotherapie, Operation und Watchful Waiting) erhalten 56,6\% der Patienten mit lokal begrenzten Prostatakarzinom eine RP, $16,4 \%$ eine Radiatio und $27 \%$ ein konservatives Vorgehen [11, 12]. In der aktuellen Follow-up-Studie dieser Patienten nach RP betrug die Inkontinenzrate $15 \%$. Die Rate der erektilen Dysfunktion betrug insgesamt $86 \%$ und bei der Subkategorie der präoperativ potenten Patienten nach nerverhaltender Operation $58 \%$ [13]. Einschränkungen des funktionellen Outcomes beeinflussen nicht nur die Lebensqualität negativ, sondern führen auch zu einem stärkeren Bedauern der Entscheidung zur Operation $[14,15]$. Entsprechend kann die Therapie der Inkontinenz und erektilen Dysfunktion entscheidend für die Lebensqualität der Patienten sein. In der Analyse der Follow-up-Patienten mit funktionellen Einschränkungen der HAROW-Studie zeigte sich, dass nur $25 \%$ der inkontinenten Patienten eine operative Therapie erhielten und nur $51 \%$ der Patienten mit erektiler Dysfunktion und Interesse an Sex, Hilfsmittel nutzen oder zumindest einmal ausprobiert haben [9]. In dieser Studie zeigte sich bereits ein Versorgungsdefizit, da die Hälfte der inkontinenten Patienten ohne Operation eine deutlich eingeschränkte Lebensqualität aufgrund ihrer Inkontinenz aufwies. Bei Patienten mit erektiler Dysfunktion und Interesse an Sex, welche noch nie Hilfsmittel zur Verbesserung ihrer erektilen Funktion genutzt hatten, berichten ein Drittel eine deutlich eingeschränkte Lebensqualität aufgrund ihrer Einschränkung. Offen 


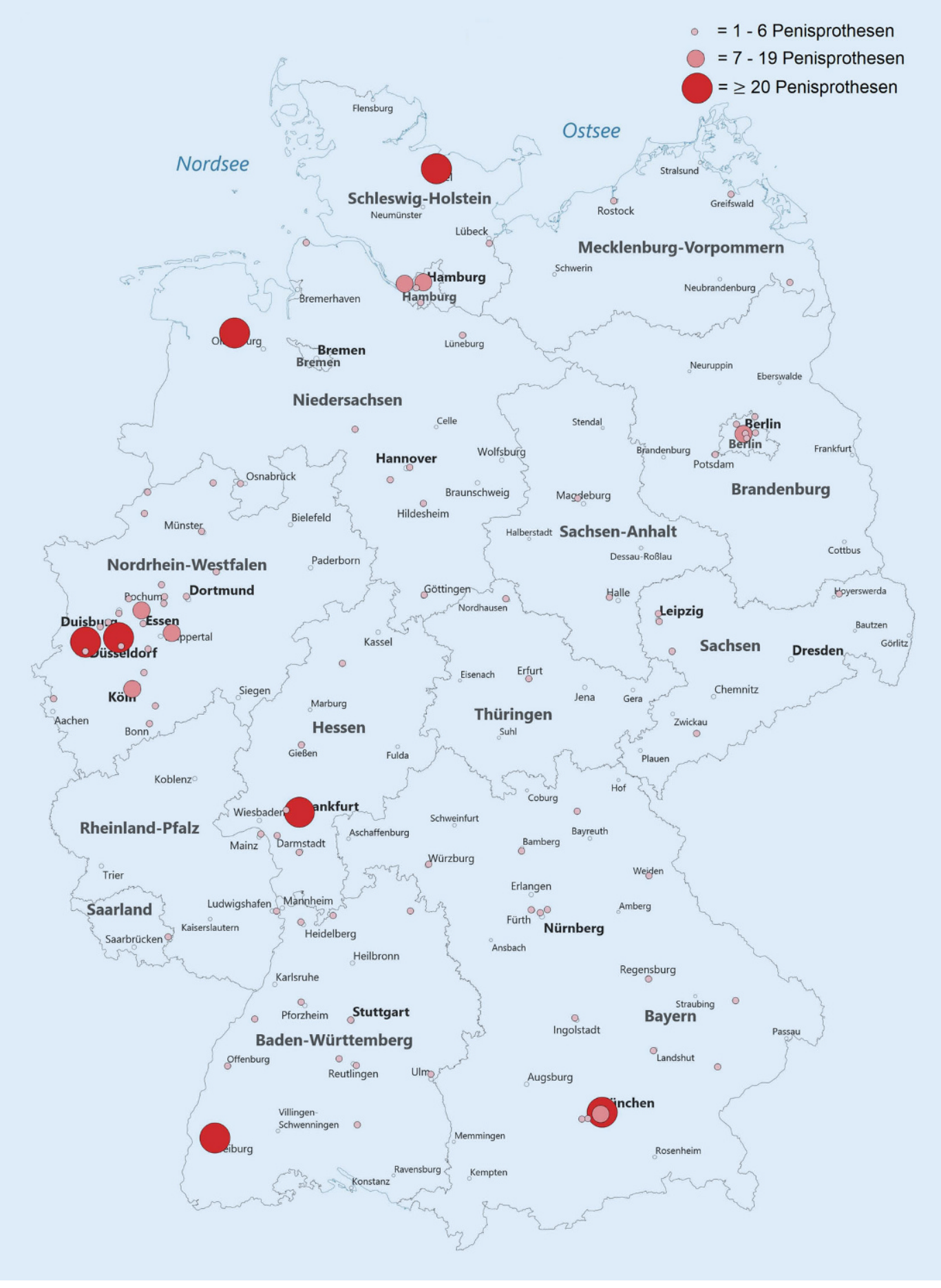

Abb. $4<$ Urologische Kliniken, die 2016 Penisprothesen implantierten (Datenquelle: Qualitätsberichtsdaten 2016, g-ba; easymap@ Kartengrafik: Eigene Darstellung)

bleiben die genauen Gründe für dieses Versorgungsdefizit. Einige Studien zeigen ein Defizit in Wahrnehmung und Kommunikation bezüglich Inkontinenz und erektiler Dysfunktion zwischen Arzt und Patient auf [16-18]. Oft ist das Internet erster Anlaufpunkt für Patienten zur Informationssuche und dem Austausch mit anderen Betroffenen [19-22].
Betrachtet man in • Abb. 5 die Anzahl von 22.500 RP im Jahr 2016 und leitet hier die oben genannte Raten an Inkontinenz und erektiler Dysfunktion ab, kann man von etwa 3300 Patienten mit Inkontinenz und 19.000 Patienten mit erektiler Dysfunktion ausgehen. Somit ergibt sich ein relevanter Bedarf an Sphinkter- und Penisprothesenimplantationen, auch wenn es bei beiden funktionellen Einschränkungen zahlreiche operative bzw. medikamentöse Therapiealternativen gibt. Unsere Studie zeigt auf, dass die Zahl der Sphinkter- und Penisprothesenimplantationen nicht mit der Zahl der RP der einzelnen Kliniken korreliert $(p=0,1$ bzw. $p=0,7)$. Dies ist ein Hinweis darauf, 


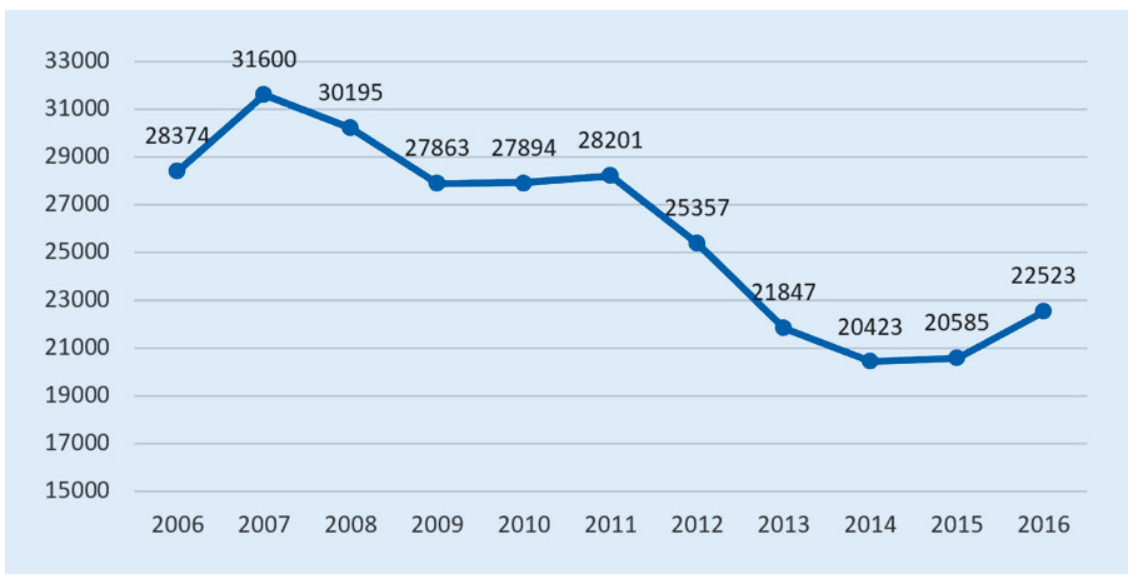

Abb. 5 × Fallzahl radikaler Prostatektomien in Deutschland

dass viele Klinik nicht die funktionellen Folgen ihrer RP selbst therapieren.

Auch andere Arbeiten ergaben bereits Hinweise auf ein Versorgungsdefizit bei der Behandlung funktioneller Einschränkungen nach RP. Eine Auswertung der SEER(Surveillance, Epidemiology, and End Results Program)-Datenbank in den USA von 16.348 Männern nach RP zeigte, dass nur $6 \%$ der Patienten eine Inkontinenzoperation erhielten. Bei einer deutlich höheren Inkontinenzrate nach RP stellt dies einen Hinweis auf eine Unterversorgung dar [23]. Eine weitere Auswertung der SEER-Datenbank zeigte, dass 2,3\% der Patienten nach RP eine Penisprothese erhalten haben [24]. Eine deutsche Studie zeigte nur eine Nutzung von $0,3 \%$ [25] und das Follow-up von 936 Patienten nach RP im Rahmen der HAROW-Studie erbrachte keinen einzigen Patienten mit Penisprothese [9].

Die einzige konstante Steigerung von 2006 bis 2016 zeigt sich im Einsatz der Penisprothese bei Geschlechtsumwandlungen. Hier verdreifachte sich die Zahl von 38 Implantationen 2006 auf 120 im Jahr 2016. Dies spiegelt die Zunahme an Geschlechtsumwandlungen in Deutschland wider [26]. Bei der Implantation von Penisprothesen bei erektiler Dysfunktion zeigt sich ab 2013 eine Stagnation, bei Sphinkterporthesenimplantationen ab 2012 sogar ein leichter Abfall. Für beide Entwicklungen kann zum einen die deutliche Abnahme von radikalen Prostatektomien ab 2011 verantwortlich sein [27]. Zum anderen konkurriert auch das ATOMS („adjustable transobturator male system") als operative Alternative zur Sphinkterprothese [28].

Bei der Implantation von Sphinkterund Penisprothesen zeigt sich eine für deutsche Verhältnisse recht ausgeprägte Zentrumsbildung. Am stärksten ist diese bei Penisprothesen ausgebildet. Hier implantieren die 7 Top-Kliniken ( $\geq 20$ Prothesen/Jahr, 7\% aller Kliniken) die Hälfte aller Penisprothesen in Deutschland. Penisprothesen werden überwiegend in Großstädten und Universitätskliniken implantiert. Bei Sphinkterprothesen implantierten die 10 Top-Kliniken ( $\geq 20$ Prothesen/Jahr, $5 \%$ aller Kliniken) ein Drittel der Sphinkterprothesen in Deutschland. Aufgrund der relevanten Komplikations- und Revisionsraten bei der urologischen Endoprothetik [4, 7] ist eine Zentrumsbildung mit entsprechend höherer operativer Erfahrung zu befürworten [5, 8]. Eine große Kohortenstudien in den USA mit 65.602 Patienten zeigte, dass selbst mit einer Erfahrung von über 200 Sphinkterprothesenimplantationen eine weitere Qualitätssteigerung möglich ist [5], wobei ein Großteil der Patienten von Urologen operiert wird, die diese Erfahrung nie erreichen [29]. Trotz der Zentrumsbildung in Deutschland wiesen 201688 \% der Kliniken mit Sphinkterprothesenimplantation $\leq 9$ Fälle/Jahr bzw. 86\% der Klinik mit Penisprothesenimplantation $\leq 6$ Fälle/Jahr auf.

In der Fallzahlentwicklung des Einsatzes der Sphinkterprothesen zeigt sich eine wesentliche Entwicklung bei Manschetten im Bereich des Blasenhalses. Hier nahm der Einsatz mit einem initialen Anteil von $29 \%$ auf $4 \%$ deutlich ab. In der Subgruppenanalyse zeigte sich ein möglicher Grund in der deutlichen Abnahme bei männlichen Patienten von $82 \%$ auf $45 \%$, während der absolute Anteil der Frauen gleichblieb (2006 39 Frauen und 177 Männer, 201621 Frauen und 17 Männer). Auch bei Penisprothesen zeigt sich eine Entwicklung des Einsatzes der beiden Typen mit einem zunehmenden Einsatz der hydraulischen Prothese, welche deutlich komfortabler für die Patienten ist [30].

Dies ist die erste Studie zur Versorgungswirklichkeit der Implantation von Sphinkter- und Penisprothesen in Deutschland. Die Nutzung von DRGAbrechnungsdaten und der Qualitätsberichte ermöglicht eine sehr genaue Darstellung der Versorgungssituation. Die Fallzahlangaben der Qualitätsberichte liegen leicht unter den Angaben der DRG-Daten, da bei den Qualitätsberichten Fallzahlen von 1-3 als 1 kodiert werden. Somit war eine exakte Differenzierung der niedrigen Fallzahlen aufgrund der Zahl an OPS-Subkategorien bei Sphinkterprothesen (3 Kategorien) nur bis mindestens 9 und bei Penisprothesen (2 Kategorien) bis mindestens 6 möglich. Die DRG-Datenbank kann nicht zwischen Fachbereichen differenzieren, sodass hier auch nicht-urologische Kliniken eingeschlossen sind. In der Analyse der Qualitätsberichte zeigt sich aber dieser Anteil (1/98 Kliniken mit Penisprothesenimplantation und 3/195 Kliniken mit Sphinkterprothesenimplantation) als sehr gering. Weiterhin erfasst die DRG-Datenbank nicht Implantationen, welche außerhalb des DRG-Systems abgerechnet wurden. In beiden Datenbanken fehlen klinische Daten, sodass weder die Indikation noch die Versorgungsqualität der Implantationen näher charakterisiert werden kann. Aufgrund der anonymen Datenquellen lässt sich beispielsweise auch nicht analysieren, ob Revisionseingriffe bei den eigenen Patienten oder möglicherweise bei zugewiesenen, extern implantierten Patienten erfolgten. Die Darstellung der Fallzahl einer Klinik im Jahr 2016 stellt überdies nur eine Momentaufnahme dar 
und kann in davor und danach liegenden Jahren variieren.

\section{Fazit für die Praxis}

- Der Versorgungsstand der urologischen Endoprothetik in Deutschland zeigt eine deutliche Zentrenbildung, aber auch eine große Zahl von Kliniken mit geringer Fallzahl.

- Da bei beiden Prothesen ein relevantes Komplikations- und Revisionsrisiko besteht, ist die operative Erfahrung entscheidend bei der Implantation.

- Seit 2012/2013 zeigt sich eine Stagnation der Fallzahlen von Penisund Sphinkterprothesenimplantationen, die in Zusammenschau mit den Prostatektomiefallzahlen eine Unterversorgung vermuten lässt.

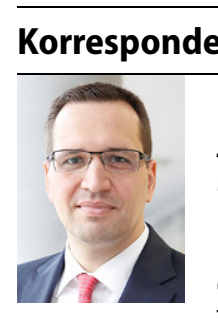

Prof. Dr. med. Dr. phil. Johannes Huber, FEBU, MHBA

Klinik und Poliklinik für Urologie, Universitätsklinikum Carl Gustav Carus an der Technischen Universität Dresden

Fetscherstraße 74, 01307 Dresden, Deutschland johannes.huber@uniklinikumdresden.de

Funding. Open Access funding enabled and organized by Projekt DEAL.

\section{Einhaltung ethischer Richtlinien}

Interessenkonflikt. M. Baunacke, C. Groeben, A. Borkowetz, A. Uhlig, M. Leitsmann, B. Volkmer und C. Thomas geben an, dass kein Interessenkonflikt besteht. J. Huber erhielt Honorare von Amgen und Janssen sowie Unterstützung für wissenschaftliche Projekte von Intuitive Surgical, Takeda und Coloplast - sämtlich außerhalb der vorliegenden Arbeit.

Für diesen Beitrag wurden von den Autoren keine Studien an Menschen oder Tieren durchgeführt. Für die aufgeführten Studien gelten die jeweils dort angegebenen ethischen Richtlinien. Datenquelle: DRGStatistik der Forschungsdatenzentren der Statistischen Ämter des Bundes und der Länder; eigene Berechnungen. easymap $\odot$ ist eine eingetragene Marke der Lutum + Tappert DV Beratung GmBH ein Unternehmen der infas Holding AG, Bonn, Deutschland.
Open Access. Dieser Artikel wird unter der Creative Commons Namensnennung 4.0 International Lizenz veröffentlicht, welche die Nutzung, Vervielfältigung, Bearbeitung, Verbreitung und Wiedergabe in jeglichem Medium und Format erlaubt, sofern Sie den/die ursprünglichen Autor(en) und die Quelle ordnungsgemäß nennen, einen Link zur Creative Commons Lizenz beifügen und angeben, ob Änderungen vorgenommen wurden.

Die in diesem Artikel enthaltenen Bilder und sonstiges Drittmaterial unterliegen ebenfalls der genannten Creative Commons Lizenz, sofern sich aus der Abbildungslegende nichts anderes ergibt. Sofern das betreffende Material nicht unter der genannten Creative Commons Lizenz steht und die betreffende Handlung nicht nach gesetzlichen Vorschriften erlaubt ist, ist für die oben aufgeführten Weiterverwendungen des Materials die Einwilligung des jeweiligen Rechteinhabers einzuholen.

Weitere Details zur Lizenz entnehmen Sie bitte der Lizenzinformation auf http://creativecommons.org/ licenses/by/4.0/deed.de.

\section{Literatur}

1. Carson CC (2020) Artificial urinary sphincter: current status and future directions. Asian J Androl 22(2):154-157

2. Averbeck MA, Woodhouse C, Comiter C, Bruschini H, Hanus T, Herschorn S et al (2019) Surgical treatment of post-prostatectomy stress urinary incontinence in adult men: report from the 6 th International consultation on incontinence. Neurourol Urodyn 38(1):398-406

3. DjakovicN,Huber J,Nyarangi-Dix J, HohenfellnerM (2010) Artificial sphincter for the treatment of incontinence. Urologe A 49(4):515-524

4. Van der Aa F, Drake MJ, Kasyan GR, Petrolekas A, Cornu JN, Young Academic Urologists Functional Urology Group (2013) The artificial urinary sphincter after a quarter of a century: a critical systematic review of its use in male nonneurogenic incontinence. Eur Urol 63(4):681-689

5. Sandhu JS, Maschino AC, Vickers AJ (2011) The surgical learning curve for artificial urinary sphincter procedures compared to typical surgeon experience. Eur Urol 60(6):1285-1290

6. Sohn M, Dietrich M, Wirthmann A, Rieger UM (2018) Reconstructive surgery in penile cancer. Urologe A 57(4):428-434

7. Scherzer ND, Dick B, Gabrielson AT, Alzweri LM, Hellstrom WJG (2019) Penile prosthesis complications: planning, prevention, and decision making Sex Med Rev 7(2):349-359

8. Henry GD, Kansal NS, Callaway M, Grigsby T, Henderson J, Noble J et al (2009) Centers of excellence concept and penile prostheses: an outcome analysis. JUrol 181(3):1264-1268

9. Baunacke M, Schmidt M, Groeben C, Borkowetz A, Thomas C, Koch R et al (2020) Treatment of postprostatectomy urinary incontinence and erectile dysfunction: There is insufficient utilisation of care in German cancer survivors. World J Urol. https:// doi.org/10.1007/s00345-020-03526-Z

10. Groeben C, Koch R, Baunacke M, Wirth MP Huber J (2016) Robots drive the German radical prostatectomy market: a total population analysis from 2006 to 2013. Prostate Cancer Prostatic Dis 19(4):412-416
11. Herden J, Ernstmann N, Schnell D, Weissbach L (2014) The HAROW study: an example of outcomes research: a prospective, non-interventional study comparing treatment options in localized prostate cancer. Urologe A 53(12):1743-1752

12. Herden J, Boedefeld EA, Weissbach L (2020) Noninvasive treatment of organ-confined prostate cancer in elderly patients-results of the HAROW study. Urologe A 59(4):450-460

13. Baunacke M, Schmidt ML, Thomas C, Groeben C, Borkowetz A, Koch R et al (2020) Long-term functional outcomes after robotic vs. retropubic radical prostatectomy in routine care: a 6-year follow-up of a large German health services research study. World J Urol 38(7):1701-1709

14. Baunacke M, Schmidt ML, Groeben C, Borkowetz A, Thomas C, Koch R et al (2020) Decision regret after radical prostatectomy does not depend on surgical approach: 6-year followup of a large German cohort undergoing routine care. J Urol 203(3):554-561

15. Steineck G, Helgesen F, Adolfsson J, Dickman PW, Johansson JE, Norlen BJ et al (2002) Quality of life after radical prostatectomy or watchful waiting. NEngl J Med 347(11):790-796

16. Borges RC, Tobias-Machado M, Gabriotti EN, Dos Santos Figueiredo FW, Bezerra CA, Glina S (2019) Post-radical prostatectomy urinary incontinence: is there any discrepancy between medical reports and patients' perceptions? BMC Urol 19(1):32

17. Litwin MS, LubeckDP, Henning JM, CarrollPR(1998) Differences in urologist and patient assessments of health related quality of life in men with prostate cancer: results of the CaPSURE database. J Urol 159(6):1988-1992

18. Rosing D, Berberich HJ (2004) Disease- and treatment related sexual disorders after radical prostatectomy. A biopsychosocial consideration. Urologe A 43(3):291-295

19. Borgmann H, Salem J, Baunacke M, Boehm K, Groeben C, Schmid M et al (2018) Mapping the landscape of urology: a new media-based cross-sectional analysis of public versus academic interest. Int JUrol 25(5):421-428

20. Baunacke M, Groeben C, Borgmann H, Salem J, Kliesch S, Huber J (2018) Andrology on the Internet: most wanted, controversial and often primary source of information for patients. Andrologia 50(2). https://doi.org/10.1111/and.12877

21. Baunacke M, Groeben C, Borgmann H, Schneider A, Kliesch S, Huber J (2016) Evaluation of the joint information platform : information offered by German urologists. Urologe A 55(7):923-932

22. Ihrig A, Karschuck P, Haun MW, Thomas C, Huber J (2020) Online peer-to-peer support for persons affected by prostate cancer: a systematic review. PatientEduc Couns 103(10):2107-2115

23. Kim PH, Pinheiro LC, Atoria CL, Eastham JA, Sandhu JS, Elkin EB (2013) Trends in the use of incontinence procedures after radical prostatectomy: a population based analysis. J Urol 189(2):602-608

24. Tal R, Jacks LM, Elkin E, Mulhall JP (2011) Penile implant utilization following treatment for prostate cancer: analysis of the SEER-Medicare database.J Sex Med 8(6):1797-1804

25. Herkommer K, Niespodziany S, Zorn C, Gschwend JE, Volkmer BG (2006) Management of erectile dysfunction after radical prostatectomy. Urologists' assessment vs patient survey responses. Urologe A 45(3):336, 8-42

26. Brunner F, Koops T, Nieder TO, Reed GM, Briken $P$ (2017) In-patient hospital care of selected sexual disorders : an analysis of data from the German 


\section{Originalien}

federal statistical office from 2000 to 2014 .

Bundesgesundheitsblatt Gesundheitsforschung Gesundheitsschutz60(9):987-998

27. Groeben C, Koch R, Baunacke M, Wirth MP, Huber J (2017) High volume is the key for improving inhospital outcomes after radical prostatectomy: a total population analysis in Germany from 2006 to 2013. World JUrol 35(7):1045-1053

28. Esquinas C, Angulo JC (2019) Effectiveness of Adjustable Transobturator Male system (ATOMS) to treat male stress incontinence: a systematic review and meta-analysis. Adv Ther 36(2):426-441

29. Lee R, Te AE, Kaplan SA, Sandhu JS (2009) Temporal trends in adoption of and indications for the artificial urinary sphincter.JUrol 181(6):2622-2627

30. Montague DK, Angermeier KW (2001) Penile prosthesis implantation. Urol Clin North Am 28(2):355-361

\section{Lesen Sie Der Urologe online auf SpringerMedizin.de}

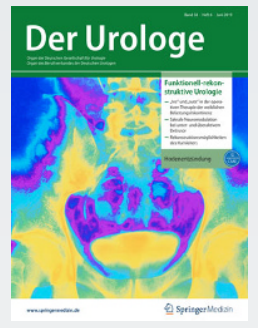

Auf SpringerMedizin.de erhalten Sie Zugang zu allen elektronisch verfügbaren Ausgaben und dem CME-Angebot Ihrer Zeitschrift - unabhängig davon, seit wann Sie Der Urologe abonniert haben. Außerdem können Sie die Zeitschrift mit dem E-Paper auch bequem auf Ihrem Tablet lesen.

> So einfach erhalten Sie Zugang zum Online Archiv Registrieren Sie sich einmalig auf www.springermedizin.de/register

Geben Sie dabei Ihre Einheitliche Fortbildungsnummer (EFN) an

- Ihr Benutzername entspricht Ihrer E-Mail-Adresse, Ihr Passwort können Sie frei wählen und später jederzeit unter "Mein Profil“ ändern.

- Falls Sie bereits ein (Print-) Abonnement bei uns haben, geben Sie bei der Registrierung die Lieferadresse Ihrer Zeitschrift an. Damit wird Ihr Abo-Zugang auf springermedizin.de freigeschaltet.
Sind Sie bereits bei SpringerMedizin.de registriert? Dann wird Ihr Zeitschriftenabonnement automatisch Ihrem Online-Nutzerkonto hinzugefügt. Sollten die Angaben Ihres Online-Accounts nicht eindeutig mit den Angaben Ihres Zeitschriften-Abonnements übereinstimmen, kann die Zuordnung nicht sicher erfolgen. In diesem Fall und bei allen anderen Fragen zum Online-Zugang kontaktieren Sie bitte unseren Kundenservice unter: Kundenservice@springermedizin.de

Telefonisch erreichen Sie die Hotline montags bis freitags von 9.00 bis 17.00 Uhr kostenfrei unter 0800-77 80777 sowie gebührenpflichtig aus dem Ausland unter $\hookleftarrow+4930884293600$ 